\title{
Eficacia y seguridad del nintedanib en fibrosis pulmonar idiopática
}

\section{Efficacy and safety of nintedanib in idiopathic pulmonary fibrosis}

Carlos Eduardo Matiz, MD. (1); Diego Fernando Pinzón Arenas, MD. (2)
(1)Médico internista, Neumólogo. Profesor asociado Medicina, Universidad El Bosque. Coordinador Comité Enfermedad Pulmonar Intersticial Difusa. Asociación Colombiana de Neumología y Cirugía de Tórax. Director CEEP.

(2)Residente de Primer Año de Medicina Interna, Pontificia Universidad Javeriana, Hospital Universitario San Ignacio. Bogotá, Colombia. Correspondencia: Carlos Eduardo Matiz, correo electrónico: carlosmatiz@hotmail.com. Recibido: 06/07/14. Aceptado: 10/09/14.

\begin{abstract}
Referencia
Richeldi L, du Bois R, Raghu G, Azuma A. Efficacy and safety of nintedanib in idiopathic pulmonary fibrosis. N Engl J Med. 2014; 370(22):2071-82.

\section{Pregunta}

En pacientes con fibrosis pulmonar idiopática ¿A dosis de 150 $\mathrm{mg}$ dos veces al día de nintedanib, hay asociación con la reducción en el decline de la capacidad vital forzada, menos episodios de exacerbaciones agudas y preservación de la calidad de vida relacionada con el estado de salud?
\end{abstract}

\section{Diseño}

Se condujeron dos ensayos clínicos fase 3 , réplicas del estudio TOMORROW (de la sigla en inglés To improve Pulmonary Fibrosis with BIBF 1129 "nintedanib"), denominados INPULSIS-1 e INPULSIS-2, de manera aleatorizada, doble ciego, controlado con placebo y en grupos paralelos.

\section{Cegamiento}

Pacientes y clínicos evaluadores.

\section{Periodo de seguimiento}

Doce meses, desde mayo de 2011 hasta septiembre de 2012.

\section{Lugar}

205 sitios, en 24 países en América, Europa, Asia y Australia.

\section{Pacientes}

1.066 pacientes asignados en los dos grupos, en un radio 3:2 respecto al placebo (INPULSIS-1 con 515 e INPULSIS-2 con 551), de 40 años o mayores, edad promedio de 66,9 años, $81 \%$ hombres, con diagnóstico de fibrosis pulmonar idiopática en los cinco años previos al estudio, con una capacidad vital forzada de $50 \%$ o más del valor predicho, difusión de monóxido de carbono (DLCO) entre 30-79\% del valor predicho, tomografía axial computarizada de alta resolución realizada en los últimos doce meses $\mathrm{y}$, de ser posible, biopsia pulmonar, estos últimos revisados por un único radiólogo y patólogo respectivamente, para verificar los criterios de elegibilidad.

Se excluyeron pacienten que venían recibiendo otras terapias para fibrosis pulmonar idiopática 
a altas dosis como prednisolona, aziatropina y $\mathrm{N}-$ acetilcisteína.

\section{Intervención}

- Se administró dosis de $150 \mathrm{mg}$ dos veces al día de nintedanib o placebo por 52 semanas.

- Seguimiento espirométrico en las semanas 2, 4, $6,12,24,36$ y 52 .

- Aplicación en las semanas 6, 12, 24 y 52 del Cuestionario Respiratorio de St. George (SRGQ), para evaluar la calidad de vida relacionada con el estado de salud.

\section{Resultados}

En el INPULSIS-1, de los 515 pacientes, 513 recibieron al menos una dosis (309 nintedanib/204 placebo), con discontinuidad del tratamiento en 78 $(25,2 \%)$ pacientes en el grupo de nintedanib y 36 $(17,6 \%)$ en el grupo del placebo (tabla 1).

En el INPULSIS-2, de 551 pacientes, 548 recibieron al menos una dosis (329 nintedanib/219 placebo), con discontinuidad del tratamiento en 78 $(23,7 \%)$ pacientes en el grupo de nintedanib y 44 $(20,1 \%)$ en el grupo placebo. En ambos grupos la presencia de algún efecto adverso fue la principal causa de no continuar en el estudio.
En ambos ensayos, INPULSIS-1 e INPULISIS-2 el cambio en la capacidad vital forzada (CVF) fue significativamente menor en el nintedanib que en el placebo, con un rango de reducción de $114,7 \mathrm{ml}$ de la capacidad vital forzada al año usando nintedanib, comparado con $239.9 \mathrm{ml}$ al año con placebo en el INPULSIS-1 y de $113,6 \mathrm{ml}$ por año y $207,3 \mathrm{ml}$ por año, respectivamente en el INPULSIS-2.

El tiempo del primer episodio de exacerbación no fue estadísticamente significativo en el grupo de INPULSIS-1, a diferencia del INPULSIS-2 en el que sí se demostró un incremento en el tiempo de presentación del primer episodio de exacerbación aguda comparado con placebo (HR 0,38; 95\% IC $0,19-0,77 ; p=0,005)$, con una menor proporción de pacientes reportados como exacerbaciones comparado con placebo (3,6\% vs. $9,6 \%)$.

No se encontraron diferencias importantes en el SGRQ respecto a cambios en la calidad de vida relacionada con el estado de la salud, en cuanto al estado basal al inicio del estudio.

Los eventos adversos fueron muy frecuentes, principalmente los síntomas gastrointestinales, con predominio de diarrea en los dos grupos que recibieron nintedanib, que a su vez fue motivo de la mayoría de retiros de pacientes durante el estudio. El compromiso hepático con elevación de enzimas

Tabla 1. Desenlaces en los grupos.

\begin{tabular}{|c|c|c|c|c|c|}
\hline \multirow[t]{2}{*}{ Desenlace } & \multicolumn{2}{|c|}{ INPULSIS-1 } & \multicolumn{2}{|c|}{ INPULSIS-2 } & \multirow{2}{*}{$\begin{array}{c}\mathbf{p} \\
<0,001\end{array}$} \\
\hline & $\begin{array}{l}\text { Nintedanib } \\
(n=307)\end{array}$ & $\begin{array}{l}\text { Placebo } \\
(n=204)\end{array}$ & $\begin{array}{l}\text { Nintedanib } \\
(n=327)\end{array}$ & $\begin{array}{l}\text { Placebo } \\
(n=217)\end{array}$ & \\
\hline Cambio desde la línea basal de CFV - ml & $-95,1$ & $-205,0$ & $-95,3$ & $-205,0$ & $<0,001$ \\
\hline $\begin{array}{l}\text { Cambio desde la línea basal de CVF del } \\
\text { predicho --- \% }\end{array}$ & $-2,8$ & $-6,0$ & $-3,1$ & $-6,2$ & $<0,001$ \\
\hline Exacerbación aguda No (\%) & $5(6)$ & 0 & & 0,03 & \\
\hline Eventos adversos & & $\mathrm{n}(\%)$ & & & \\
\hline Cualquier evento adverso & $298(96,4)$ & $181(88,7)$ & $311(94,5)$ & $198(94,4)$ & \\
\hline Diarrea & $190(61,5)$ & $38(18,6)$ & $208(63,2)$ & $40(18,3)$ & \\
\hline Náusea & $70(22,1)$ & $12(5,9)$ & $86(12,1)$ & $16(7,3)$ & \\
\hline Tos & $47(15,2)$ & $26(12,7)$ & $38(11,6)$ & $31(15,5)$ & \\
\hline Vómito & $40(12,9)$ & $4(2,0)$ & $34(10,3)$ & $7(3,2)$ & \\
\hline Bronquitis & $36(11,7)$ & $28(13,7)$ & $31(9,4)$ & $17(7,8)$ & \\
\hline
\end{tabular}


hepáticas estuvo presente en una alta proporción de pacientes; en INPULSIS-1 15 (4,9\%) y en INPULSIS-2 $17(5,2 \%)$.

\section{Conclusión}

En pacientes con fibrosis pulmonar idiopática, nintedanib reduce el decline en la capacidad vital forzada, disminuyendo la progresión de la enfermedad. Hubo diferencia significativa a favor del nintedanib para el tiempo de exacerbación y los cambios en el SGRQ en el INPULSIS-2 pero no en el INPULSIS-1.

Los eventos adversos fueron comunes en ambos ensayos, principalmente la presencia de diarrea; sin embargo la mayoría de pacientes continuó recibiendo nintedanib durante el período de tratamiento.

\section{Comentario}

Aprovechando el entendimiento de los mecanismos fisiopatólogicos de la fibrosis pulmonar idiopática, relacionados con los cambios aberrantes en la proliferación de tejido fibroso (1) y la intervención de una variedad de receptores de tirosinquinasa en los que intervienen factores de crecimiento como el derivado de plaquetas, el fibroblástico y vascular (2), nuevos medicamentos como el nintedanib, han surgido como innovaciones para el tratamiento de una enfermedad de la que existe poca intervención farmacológica.

Desde el estudio TOMORROW en fase de ajuste de dosis, se observaron beneficios de este medicamento, que sugerían intervención sobre la progresión de la enfermedad y la mejor calidad de vida de los pacientes que la padecían (3). INPULSIS-1 e INPULSIS-2 confirmaron este primer dato, pues demostraron menor progresión en la disminución de la capacidad vital forzada a un año.

Sin embargo, conviene mencionar que el estudio fue realizado en pacientes con compromiso leve a moderado, lo que indicaría el inicio de tratamiento en fases tempranas de la enfermedad, aspecto que debe ser evaluado en nuevos estudios, y que adicionalmente gran número de la población estudiada presentó efectos secundarios, principalmente diarrea, que pueden intervenir en la continuidad del tratamiento y la adherencia al mismo.

Por otro lado, sería importante la evaluación de la mortalidad a largo plazo, ya que no fue factor tenido en cuenta como desenlance en este estudio. Será relevante para estudios futuros determinar si tal disminución de la progresión del deterioro de la función pulmonar, actúa de igual forma en la intervención de la mortalidad.

\section{Conflictos de intereses}

Los autores declaran no tener conflictos de interés.

\section{Bibliografía}

1. King Te Jr, Pardo A, Selman M. Idiopathic pulmonary fibrosis. Lancet 2011;378:1949-61.

2. Chaudhary NI, Roth GJ, Hilberg F, Müller-Quernheim J, Prasse A, Zissel G. Inhibition of PDGF, VEGF and FGF signaling attenuates fibrosis. Eur Respir J. 2007;29:976-85.

3. Richeldi L, Costabel U, Selman M, Kim DS, Hansell DM, Nicholson AG. Efficacy of a tyrosine kinase inhibitor in idiopathic pulmonary fibrosis. N Engl J Med. 2011;365:1079-87. 